Review www.pjkd.com.pk

\title{
Histologic Variants of FSGS: An epidemiological and pathological perspective
}

\author{
Shaheera Shakeel, Rahma Rashid, Muhammed Mubarak
}

Javed I. Kazi Department of Histopathology, Sindh Institute of Urology and Transplantation (SIUT), Karachi, Pakistan

\begin{abstract}
Focal segmental glomerulosclerosis (FSGS) is a conglomerate of glomerular pathological lesions unified by the segmental distribution of proliferative, sclerosing or collapsing lesions in less than $50 \%$ of glomeruli (i.e., focal distribution) in the early stages of the process and can only be diagnosed on renal biopsy. Epidemiologically, FSGS has acquired the top position among the causes of nephrotic syndrome (NS) in adults in many parts of the world and has emerged as the main cause of chronic kidney disease (CKD) and end-stage renal disease (ESRD) worldwide. Numerous approaches have been used to classify this lesion, but all have merits and demerits and no one system is entirely satisfactory. One of the popular schemes for the classification is based on morphological features and distribution of the lesions in the glomeruli, the so-called Columbia classification of FSGS. In this review, we briefly summarize the relevant epidemiology and pathology of FSGS variants in the light of our own experience with the use of this classification.
\end{abstract}

Key words: $F S G S$, histological classification, nephrotic syndrome, end-stage renal disease, segmental

\section{Corresponding author:}

Prof. Dr. Muhammed Mubarak

Javed I. Kazi Department of Histopathology,

Sindh Institute of Urology and Transplantation,

Dewan Farooq Medical Complex (DFMC), Chand Bibi Road,

Karachi-74200, Pakistan,

Email address: drmubaraksiut@yahoo.com

Received: January 22,2021. Accepted March 08, 2021.

PJKD 2021;5(1):2-10

\section{Introduction}

Focal segmental glomerulosclerosis (FSGS) is a morphologically defined glomerulopathic lesion, which presents with divergent clinical manifestations, exhibits variable clinical course and progresses to endstage renal disease (ESRD) in a sizeable proportion of patients in both adult and pediatric populations. ${ }^{1}$ This heterogeneous paradigm of FSGS encompasses various etiopathogenetic pathways leading to the hallmark features of segmental implosion/expansion/sclerosis of capillary tufts and accumulation of extracellular matrix together with variable changes in podocyte phenotypes in majority of cases. FSGS accounted for $39.87 \%$ and $38.14 \%$ of cases presenting with nephrotic syndrome (NS) in our adult and pediatric populations, respectively.

\section{Histological Classification Initial Concept:}

The heterogeneity of the lesion demands its classification as in all diseases. Numerous attempts have been made in the past to classify FSGS, but no perfect classification system has been devised yet. The classification framework proposed by Barisoni et al. is based on two premises including etiologic factors and glomerular morphologic appearance with podocytes phenotype. ${ }^{5}$ They also suggested that the initiating cascade of podocyte injury and consequent death is more severe in FSGS as compared to minimal change disease (MCD) and in some cases, this podocyte injury may lead to variable amount of 
Histological variants of FSGS

podocyte proliferation. Interestingly, this structural alteration in podocyte shape is reversible with steroid therapy in MCD but it is irreversible and progressive in FSGS. ${ }^{6}$ Furthermore, they also advocated that whenever possible, diagnosis of podocytopathies should include morphologic entity accompanied by etiologic and pathogenetic mechanisms. ${ }^{5,6}$

Table 1: Histologic variants of Focal Segmental Glomerulosclerosis.

\begin{tabular}{|ccc|}
\hline FSGS Histologic Variants (\%) \\
\hline$\bullet$ & Not otherwise specified (NOS) & $(42-77 \%)$ \\
& (formerly called classic FSGS) \\
$\bullet$ & Collapsing variant. $\quad(2-36.6 \%)$ \\
$\bullet$ & Tip variant. & $(9.8-1.4 \%)$ \\
$\bullet$ & Perihilar variant. & $(1.1-26 \%)$ \\
$\bullet$ & Cellular variant. & $(0.5-25.5 \%)$ \\
\hline
\end{tabular}

\section{Genetic Mutations and Structural Changes:}

During the last few 3-4 decades, the discovery of mutations of podocyte genes and their association with genetic variants of FSGS has produced detailed analytic insight into podocyte taxonomy and its role in better management of these patients. ${ }^{7}$ Several genetic mutations have been identified; of these, nephrin and podocin are most frequently observed. These podocyte genes encode structural proteins or enzymes, which are actively engaged in signaling mechanisms that regulate podocyte growth, differentiation, motility and cell-to-cell interactions. ${ }^{8.9}$ Any dysregulation in this signaling event, results in structural disarray and condensation of actin cytoskeleton and foot process effacement. This stereotyped response to injury would ultimately, in severe cases, lead to podocyte detachment from the glomerular basement membrane (GBM). ${ }^{7.8}$ This denuded area of GBM comes in contact with the parietal epithelium and begins the sequence of events leading to synechiae formation, which are thought to be the earliest committed lesions in the development of FSGS. The complex structural podocyte composition is maintained by sophisticated metabolic environment and factors that endow very rigid or dynamic cytoskeletal changes pose threats to podocyte' $\mathrm{s}$ survival. ${ }^{7.9}$ In addition to podocytes, other glomerular cells including mesangial cells, endothelial cells and parietal epithelial cells are also involved in either the pathogenesis or progression of the disease. ${ }^{10}$

\section{Columbia Classification}

The Columbia classification has described five histologic subtypes of FSGS: not otherwise specified (NOS), tip, cellular, perihilar and collapsing variant. ${ }^{11}$ Several studies have been published during recent past elaborating the incidence, clinical course and progression of disease according to these variants in both adults and children. ${ }^{11,12}$ We have also earlier reported relative frequencies and clinicopathologic features of these variants among both pediatric and adult nephrotic populations. ${ }^{13,14}$

\section{Not Otherwise Specified (NOS)}

The prevalence of FSGS, NOS variant varies from $42 \%$ to $77 \%$ of all cases of FSGS and is the predominant lesion among all the five variants in most of the studies from different ethnic and geographic areas. ${ }^{13-18}$ FSGS, NOS variant is a heterogeneous subtype resulting from various glomerular and non-glomerular diseases; in fact, it is a collage of a variety of glomerular alterations that culminate in a common morphologic pattern. ${ }^{15,16}$ In our cohorts of children and adults, this variant accounted for $89.1 \%$ and $76.6 \%$, respectively. ${ }^{13,14}$ This variant does not have specific distinguishing features and does not meet the diagnostic criteria for all other four variants (Figure 1). It is also the most common lesion observed in repeat renal biopsies in several studies. In fact, it represents the diagnosis of exclusion in the hierarchical classification of FSGS. 

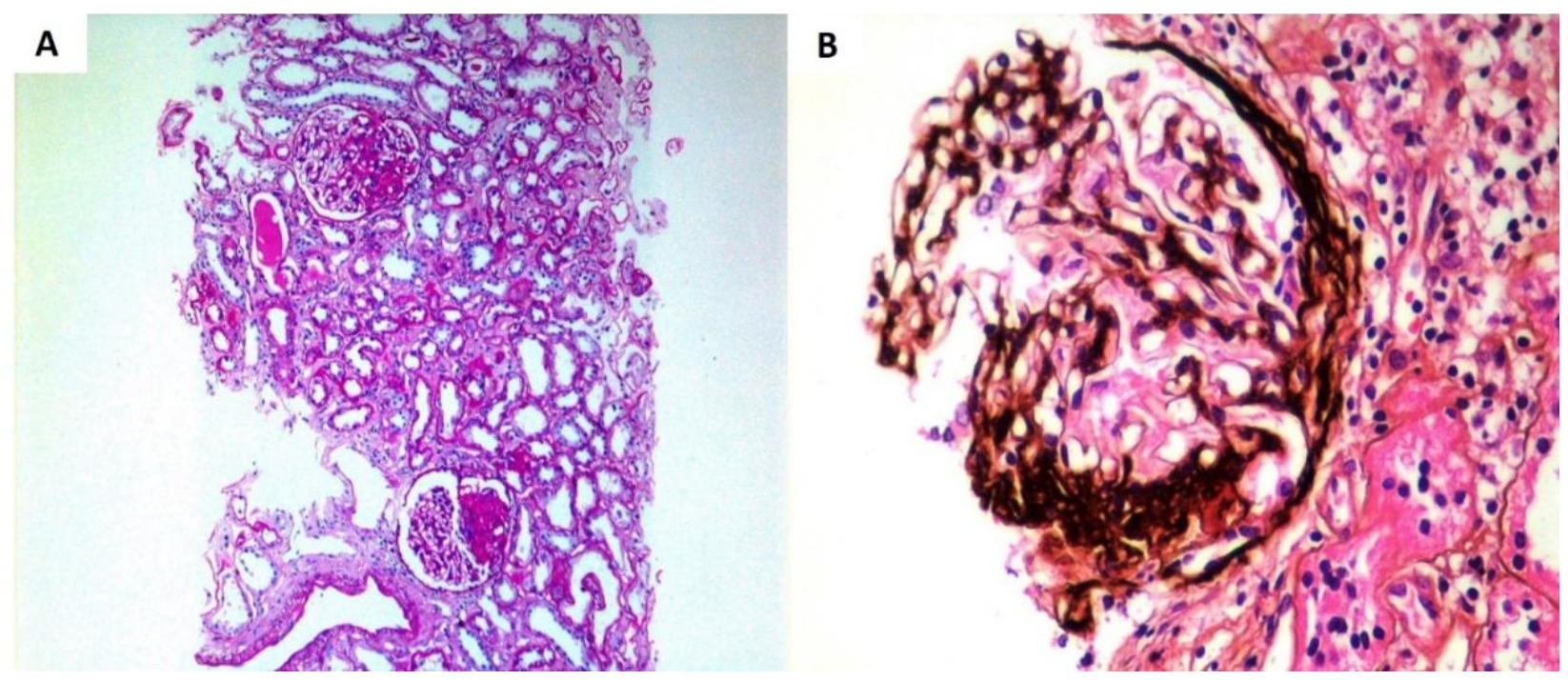

Figure 1. FSGS, NOS variant. (A) Two glomeruli showing segmental scarring of non-descript location and adhesion formation with Bowman' s capsule. Mild patchy tubular atrophy is seen. (PAS, x200). (B) Silver stain showing one glomerulus with segmental scarring in the lower portion of the tuft with adhesion formation. (Jones Silver stain, $\times 400$ ).

\section{Collapsing Variant}

The second most common variant in most of the studies is the collapsing variant. The relative frequency of collapsing variant is highly variable ranging from 2 to $36.6 \%$ in different studies. In our series, this lesion accounted for $8 \%$ and $12 \%$ of cases in pediatric and adult nephrotic cohorts, respectively. ${ }^{13,14}$

This important variant may be primary or secondary to some viral etiology including human immunodeficiency virus (HIV), parvovirus B19, polyoma virus, Epstein-Barr virus (EBV) or cytomegalovirus (CMV), drug reactions, vasoocclusive disease or chronic allograft nephropathy. ${ }^{19}$ More recently, a number of cases of collapsing variant of FSGS have been reported in association with novel coronavirus disease, COVID-19, particularly in patients of African American/black origin. ${ }^{20}$ This lesion was first recognized as HIV-associated nephropathy (HIVAN) in HIV-positive patients with poor prognosis, but, later on, it was established that the clinical presentation in patients with negative HIV serology may be markedly variable ranging from mild proteinuria to severe proteinuria and deranged renal functions. ${ }^{21,22}$

More recent findings have led to the suggestion that collapsing glomeulopathy (CG), another term used to denote the lesion, may be considered as a distinct clinicopathological entity. The salient morphologic features of collapsing variant are implosive collapse of glomerular capillary tuft with marked occlusion or sub-occlusion of capillary lumena and remarkable extracapillary hypercellularity (Figure 2). These proliferating cells appear to originate from visceral epithelial cells but they are phenotypically distinct from mature podocytes. ${ }^{5,7}$

The morphologic clue to suggest their origin from visceral epithelium is their attachment with glomerular basement membrane or centripetal location within the Bowman' $\mathrm{s}$ space where they are found contiguous with the identifiable podocytes. ${ }^{7,23}$ The mature podocytes do not proliferate and do not express maturity markers like vimentin, WT-1, CALLA, GLEPP-1, synaptopodin, c3b receptor, podocalyxin and PHM-5., ${ }^{7,923}$ However, the diagnostic utility of these immunohistochemical markers to distinguish FSGS variants needs further substantiative large-scale studies. 


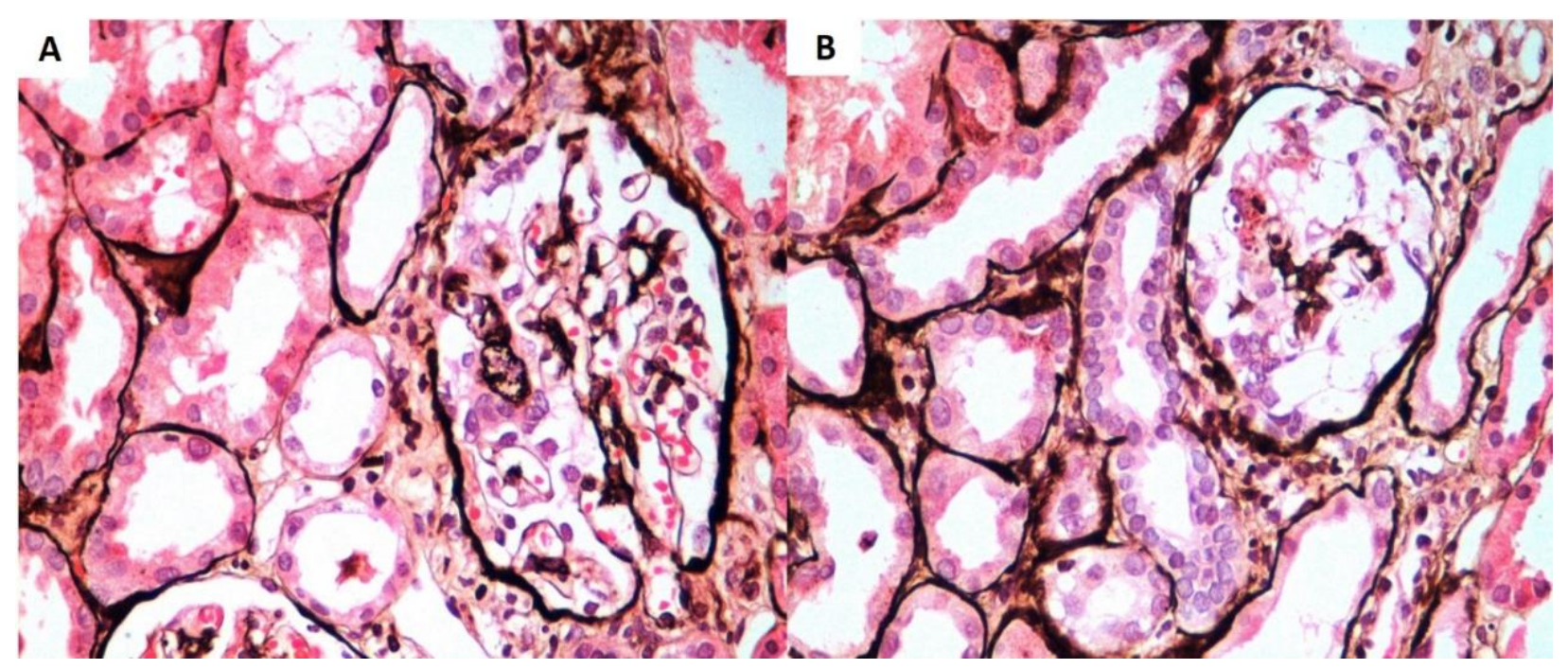

Figure 2. FSGS, collapsing variant. (A) A glomerulus showing segmental collapse of capillary tufts with associated podocyte hyperplasia. (Jones Silver stain, $\times 200$ ). (B) Another glomerulus showing global collapse of capillary tufts with marked podocyte proliferation forming pseudocrescent. Mild tubular atrophy is seen in the background. (Jones Silver stain, $\times 200$ ).

Some recent studies have also shown that origin of cells proliferating in the Bowman' s space in collapsing lesion is debatable and some investigators have argued that positivity of parietal cell markers suggest possible origin of most of these proliferating cells from parietal epithelial cells rather than the podocytes. ${ }^{9,19}$ Furthermore, some progenitor cells expressing stem cell markers including CD133 and CD24 may also serve as reserve cells to restore vanished podocytes. The hypertrophy of glomeruli, which itself is an outcome of increased single nephron glomerular filtration rate, further aggravates the mismatch between the decreased quantity of podocytes and glomerular basement membrane and leads to propagation of injurious process.

A few experimental animal model studies have shown that the toxins like diphtheria toxin (DT) or pseudomonas exotoxin A, that target podocytes specifically and exclusively are capable to cause the morphologic appearance of FSGS. ${ }^{23}$ Loss of more than $40 \%$ of podocytes leads to overt FSGS with clinical symptoms of high-grade proteinuria and renal insufficiency, indicating worsening of disease. The initial podocyte loss is followed by secondary autonomous wave of neighboring podocyte injury which might escape the initial insult. The mediators responsible for this secondary wave of podocyte injury are not yet well recognized but hypothetically they might decrease podocyte survival factors or might increase noxious factors, such as shear stress, angiotensin II, or transforming growth factor- $\beta$ (TGF- $\beta$ ). The overexpression of TGF $\beta$ or its downstream proteins, the 'Smads' , or activation of the renin-angiotensin system, which upregulates TGF $\beta$, play pivotal role in further progression of the disease. $^{19,23}$

Another contributing factor in the progression of FSGS in addition to glomerulosclerosis is the progressive involvement of tubulointerstitial compartment. Although this compartment may be involved in any histologic variant of FSGS with variable degrees of severity but its dramatic involvement is readily described in collapsing variant of FSGS, indicating the notorious clinical course of the disease. The leakage of proteins from glomerular filtration barrier (GFB) due to loss of podocytes into the tubular lumena initiates the cascade of injurious processes which ultimately leads to irreversible damage to tubulointerstitial compartment. ${ }^{12,24}$ The cytokines such as TGF- $\beta$, when present in the tubules, will 
Histological variants of FSGS

recruit inflammatory cells, which in turn, produce and release other cytokines, including interleukin-1 (IL-1), tumor necrosis factor alpha (TNF- $\alpha$ ), and other chemokines. This inflammatory infiltrate leads to mesangial matrix deposition, promoting the collapse of glomeruli as well as damages tubular epithelial cells, and some tubular epithelial cells might undergo transformation to mesenchymal cells. These mesenchymal cells, as well as recruited and stimulated fibroblasts, result in production and deposition of collagenous matrix and become a clinical insignia of progressive loss of renal function in the form of interstitial fibrosis. ${ }^{23}$

\section{Tip Variant}

The next variant of FSGS is tip variant, which accounted for $9.8 \%$ and $1.4 \%$ of cases among our adult and pediatric nephrotic cohorts. ${ }^{13,14}$ The prevalence of this lesion also varies widely from $4.8 \%$ to $37 \%$ in adult population in different studies. This lesion was first introduced by Howie and Brewer and later it was acknowledged and included in the Columbia classification schema of FSGS. According to defining criteria, a glomerulus with tip variant, on light microscopy, reveals a conglomeration of morphological changes at the characteristic topographic location of tubular pole and it needs definite identification of vascular pole of the involved glomerulus (Figure 3).

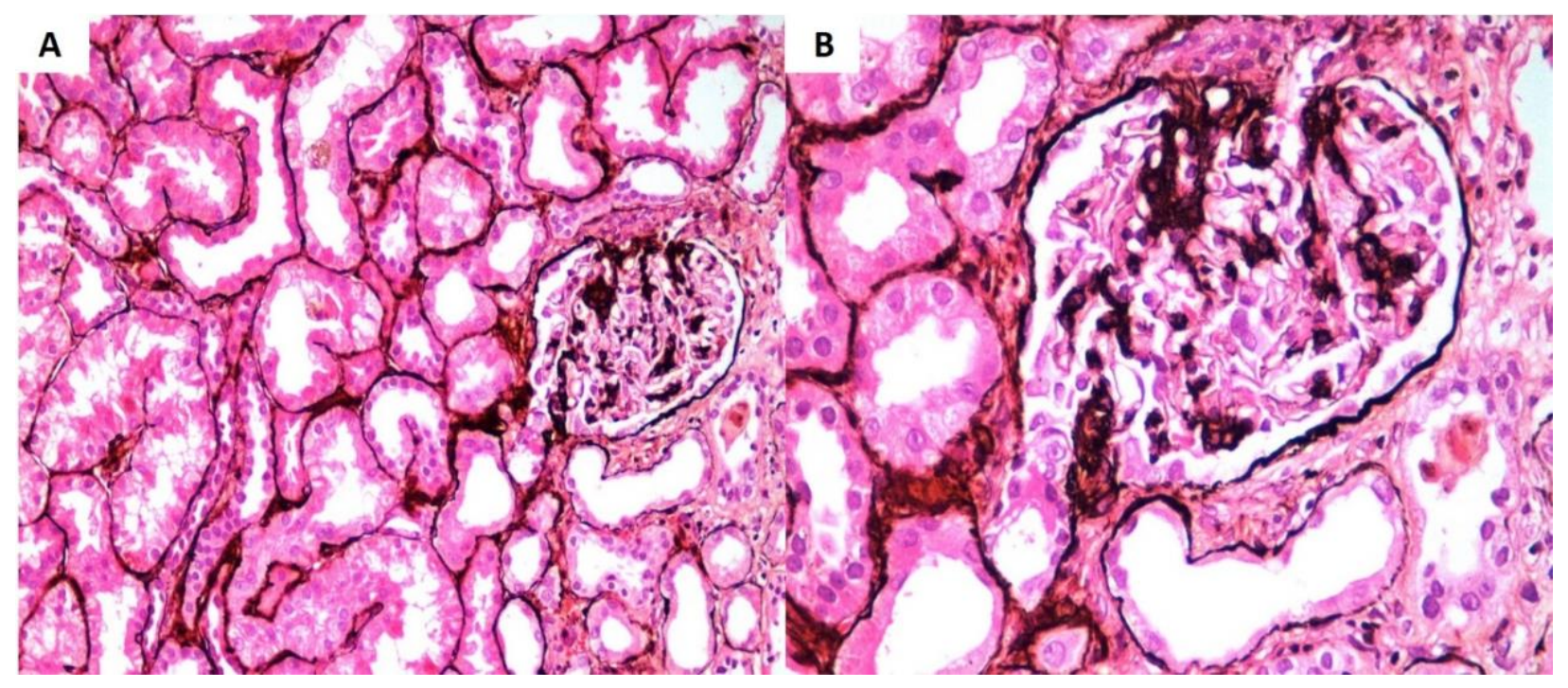

Figure 3. FSGS, tip variant. (A) Low-power photomicrograph showing a glomerulus with adhesion formation at the tubular pole. Mild tubular atrophy is seen in the background. (Jones Silver stain, $\times 200$ ). (B) The same glomerulus as shown in A with segmental sclerosis and adhesion formation at the tubular pole. (Jones Silver stain, $\times 400)$.

There might be segmental occlusion of capillary tufts or segmental expansion of capillary tufts with intracapillary foam cells with hypertrophy of overlying podocytes bridging towards parietal epithelial cells and proximal tubular epithelial cells with or without herniation into proximal tubule. ${ }^{1,11}$ In some instances, synechiae formation between the glomerular tuft and Bowman' s capsule at tubular neck or the confluence of podocytes with parietal or tubular epithelial cells at the tubular neck might be observed. In view of above morphologic appearances, collapsing variant should be excluded.

This lesion might be very subtle, so conscious evaluation of multiple serial sections of the biopsy must be examined, so that early lesions are not missed. This might be the reason behind reported low frequency of this lesion from different parts of the world..$^{12,19}$ The early description of glomerular tip lesion was not only confined to FSGS, rather it reflects a curious glomerular abnormality that might be 


\section{Histological variants of FSGS}

seen independently in heterogenous proteinuric renal glomerulopathies including membranous glomerulopathy or even in diabetic glomerulosclerosis.

Tip lesion usually presents clinically with very heavy proteinuria and there are some controversies among several authors regarding the existence of this unique lesion. Some authors suggest that it is simply a response of glomerulus to heavy proteinuria in the absence of any other abnormality on light microscopy and it may represent one form of MCD but others postulate that it is a well-defined and established specific pathologic entity. ${ }^{12,14}$ A few studies have observed that tip lesion follows more favorable clinical course and response to steroid treatment, which is comparable to that of MCD, while others demonstrate that clinical course and outcome is similar to that of patients with FSGS, NOS variant and question the clinical significance of this variant. ${ }^{12,25}$ Light microscopy and even electron microscopy is not helpful in this regard because it cannot discriminate between the two lesions. Although some authors suggest that immunohistologic evaluation of podocyte protein expression might be helpful because this protein expression is altered in FSGS but not in MCD. ${ }^{9,12,23}$

Arias et al, have observed progression to chronic kidney disease (CKD) and even to end-stage renal disease (ESRD) in $19.2 \%$ of patients with tip lesion and they proposed that tip variant should not be considered as a prognostically favorable disease; rather, it could be an early morphologic insignia of severe glomerular disease. ${ }^{26,27}$ Similar observations were made by Howie et al who have proposed that glomerular tip lesion may progress to NOS lesion and it should not be considered as a benign disease. ${ }^{28}$

Conclusively, glomerular tip lesion is a distinct morphologic pattern of FSGS which usually exhibits more favorable clinical course in terms of remarkable response to steroid therapy but in long-term follow up studies, tip lesion may progress to CKD and even to ESRD. ${ }^{14,26-29}$ These observations question the distinctness of this entity from NOS variant. As we have discussed earlier, only the clinical course and response to treatment can predict the biological behavior of this unique lesion.

\section{Perihilar Variant}

Perihilar variant accounted for $1.1 \%$ and $0.7 \%$ of our adults and children with NS, respectively. ${ }^{13,14}$ Perihilar variant is commonly observed in patients with segmental sclerosing lesions mediated by an adaptive response to hyperfilteration or nephron loss or glomerular hypertension. Howie et al have observed that the lesion found in cases with secondary FSGS was almost always perihilar and this appearance is helpful in distinguishing primary from the secondary forms in the absence of clinical data. $^{29}$

The relative frequencies of perihilar variant reported in literature vary widely ranging from $1.1 \%$ to $26 \%$, reiterating the possible role of various environmental, infectious as well as socioeconomic etiopathogenetic factors underlying the pathogenesis of this variant. ${ }^{12,15-18}$ The defining histologic features of perihilar variant are presence of perihilar hyalinosis with or without segmental sclerosis (Figure 4). Furthermore, among these involved glomeruli, more than $50 \%$ sclerotic lesions should be in the perihilar region to designate it as perihilar variant. Other glomeruli may show morphologic features of NOS variant but they should be present as a minor component. The presence of any single glomerulus with hypercellular or collapsing lesion excludes the perihilar variant, so careful evaluation is required for exclusion of these lesions, while presence of tip lesion does not exclude the diagnosis of perihilar variant. 


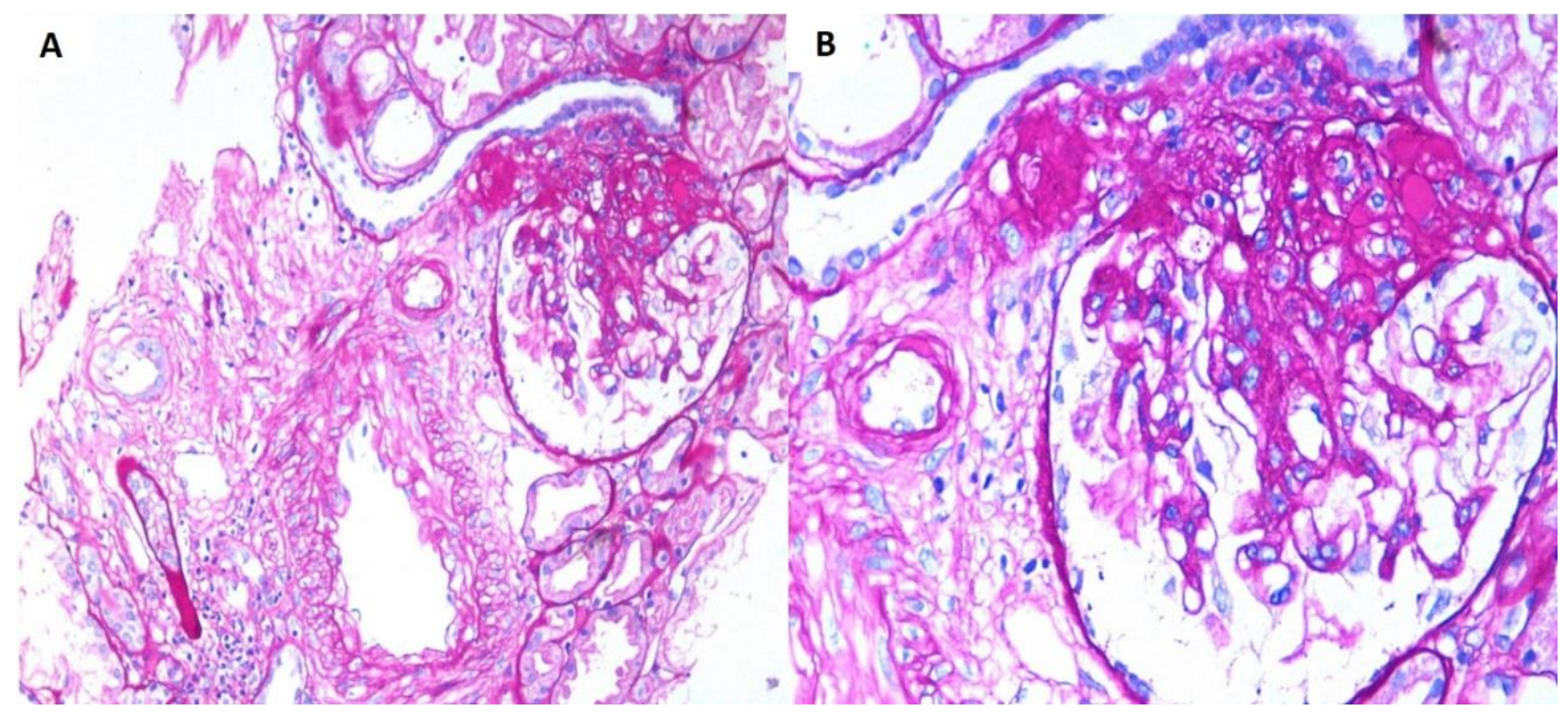

Figure 4. FSGS, perihilar variant. (A) Low-power photomicrograph showing a glomerulus with segmental scarring and hyalinosis involving the hilar region. Mild tubular atrophy is seen in the background. (PAS, $\times 200)$. (B) The same glomerulus as shown in A with segmental sclerosis and hyalinosis at the hilar pole. (PAS, $\times 400$ ).

In addition, there might be some degree of mesangial hypercellularity, lipid vacuoles in the cytoplasm of and hypertrophy/ hyperplasia of podocytes, although the later phenomenon is infrequent in this variant as compared to other ones. ${ }^{16,25,26}$ As far as clinical course and progression of disease is concerned, some authors have observed that majority of patients with perihilar variant present with sub-nephrotic range proteinuria and $82 \%$ of the patients on follow-up progress to CKD. ${ }^{25,29}$ In some studies, there were very few number of cases with perihilar variant, so statistical analysis could not be performed. ${ }^{25}$ Some authors postulate that this disease has an insidious onset and slow progression toward CKD and this variant is frequently observed in patients with secondary causes of FSGS.

\section{Cellular Variant}

The last, but not the least, variant is very unique morphologically, i.e., the cellular variant. We observed this variant in $0.5 \%$ of adult nephrotic patients and $0.7 \%$ of pediatric patients. ${ }^{13,14}$ The reported frequency of this variant is also divergent ranging from 0.5 to $25.5 \%$ in different studies. ${ }^{14,30}$ In most studies, however, the prevalence of this variant is markedly low. The defining histologic features for this variant include the presence of at least one glomerulus with segmental occlusion of capillary lumina by endocapillary proliferation of foam cells and/or macrophages and/or endothelial cells and/or neutrophils and/or lymphocytes with or without karyorrhectic debris or fibrin. ${ }^{31,32}$

In contrary to the primary definition of cellular variant, the Columbia classification also expanded the defining criteria to include the proliferation of extracapillary cells, particularly podocytes and it should not be accompanied by segmental collapse of capillaries; if later is present, the lesion would qualify for collapsing variant (Figure 5). In addition, hyalinosis, segmental scarring or synechiae are not features of this remarkable histologic variant. ${ }^{11,12,15}$ Keeping in mind all these restrictive criteria, one should vigorously search to exclude other variants of FSGS. In addition, a careful interpretation of clinical manifestations, serological findings and immunoflourescence results is mandatory for proper categorization of this lesion. These limitations might be explanatory behind the very low reported frequency of this variant. 

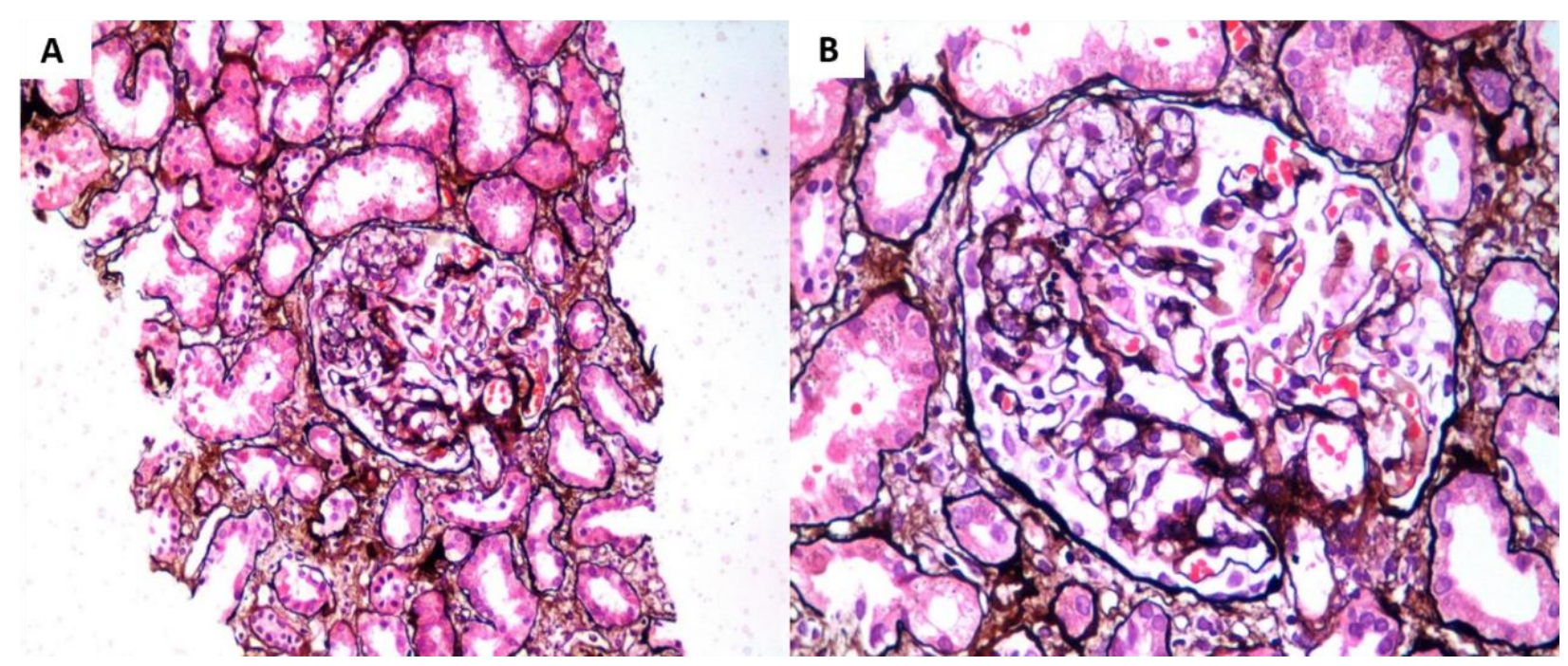

Figure 5. FSGS, cellular variant. (A) Low-power photomicrograph showing a glomerulus with expansile lesion. Mild tubular atrophy is seen in the background. (Jones Silver stain, $\times 200$ ). (B) The same glomerulus as shown in A with segmental expansion of tufts near the tubular pole. Capillary loops are distended with intracapillary foam cells and inflammatory cells. Mild podocyte hyperplasia is seen. Mild tubular atrophy is seen. (Jones Silver stain, $\times 400)$.

The clinical course and prognosis of this rare variant are also not very clear as yet due to scarce number of patients observed in the literature; however, Stokes et al. have observed that there is no significant difference in clinical course as well as the outcome of these patients from the NOS variant. ${ }^{32}$

\section{Conclusion}

In conclusion, FSGS is one of the leading causes of CKD and ESRD. In fact, FSGS is a morphologic diagnosis and it does not actually indicate a single disease entity. Acknowledging that the common pathogenetic element among the heterogenous variety of lesions in FSGS is the podocyte injury, some authors propose that the various clinical and histologic variants are the result of variable podocyte response to injurious stimuli: effacement, apoptosis, arrested differentiation, or dedifferentiation. Additional detailed insights into the pathogenesis as well as the novel genetic markers may further enhance our understanding about the heterogeneity of the variants of this morphologic entity.

\section{References:}

1. D'Agati VD. Pathobiology of focal segmental glomerulosclerosis: new developments. Curr Opin Nephrol Hypertens. 2012;21(3):243-50.

2. Kazi JI, Mubarak M, Ahmed E, Akhter F, Naqvi SA, Rizvi SA. Spectrum of glomerulonephritides in adults with nephrotic syndrome in Pakistan. Clin Exp Nephrol. 2009;13(1):38 - 43.

3. Mubarak M, Lanewala A, Kazi JI, Akhter F, Sher A, Fayyaz A, et al. Histopathological spectrum of childhood nephrotic syndrome in Pakistan. Clin Exp Nephrol. 2009;13:589 - 93.

4. Lanewala A, Mubarak M, Kazi JI, Akhter F, Sher A, Fayyaz A, et al. Treatment and prognosis of primary focal segmental glomerulosclerosis in children from Pakistan. Saudi J Kidney Dis Transplant. 2012;23:513-20.

5. Barisoni L, Kriz W, Mundel P, Agati VD. The dysregulated podocyte phenotype: a novel concept in the pathogenesis of collapsing idiopathic focal segmental glomerulosclerosis and HIV-associated Nephropathy. JASN 1999:10:51-61.

6. D Agati V. The many masks of focal segmental glomerulosclerosis. Kidney Int. 1994;46(4):1223-41.

7. Barisoni L, Schnaper HW, Kopp JB. A proposed taxonomy for the podocytopathies: a reassessment of the primary nephrotic diseases. Clin J Am Soc Nephrol. 2007;2(3):529-42.

8. Pavenstädt H, Kriz W, Kretzler M. Cell Biology of the Glomerular Podocyte. Physiological Reviews. 2003;83: 253-307. 


\section{Histological variants of FSGS}

9. Fan Q, Xing Y, Ding J, Guan N, Zhang J. The relationship among nephrin, podocin, CD2AP, and alpha-actinin might not be a true 'interaction' in podocyte. Kidney Int. 2006;69:1207-15.

10. St John PL, Abrahamson DR: Glomerular endothelial cells and podocytes jointly synthesize laminin-1 and -11 chains. Kidney Int. 2001; $60: 1037$ - 1046.

11. Deegens JK, Steenbergen EJ, Borm GF, Wetzels JF. Pathological variants of focal segmental glomerulosclerosis in an adult Dutch population - epidemiology and outcome. Nephrol Dial Transplant. 2008;23(1):186-92.

12. Thomas DB. Focal Segmental Glomerulosclerosis: A Morphologic Diagnosis in Evolution. Archives of Pathology \& Laboratory Medicine: February 2009, Vol. 133, No. 2, pp. 217-223.

13. Shakeel S, Mubarak M, Kazi JI. Frequency and clinicopathological correlations of histopathological variants of pediatric idiopathic focal segmental glomerulosclerosis. Indian J Nephrol. 2014; 24(3): 148 - 153.

14. Shakeel S, Mubarak M, Kazi JI, Jafry N, Ahmed E. Frequency and clinicopathological characteristics of variants of primary focal segmental glomerulosclerosis in adults presenting with nephrotic syndrome. J Nephropathol. 2013; 2(1): $28-35$.

15. D' Agati V. Pathologic classification of focal segmental glomerulosclerosis. Sem Nephrol . 2003;23:117 - 34.

16. D' Agati V, Fogo AB, Bruijin JA, Jennette JC. Pathologic classification of focal segmental glomerulosclerosis: a working proposal. Am J Kidney Dis . 2004;43:368 - 82.

17. Deegens JK, Steenbergen EJ, Borm EF, Wetzels JFM. Pathological variants of focal segmental glomerulosclerosis in an adult Dutch population-epidemiology and outcome. Nephrol Dial Transplant . 2008;23:186 - 92.

18. Nada R, Kharbanda JK, Bhatti A, Minz RW, Sakhuja V, Joshi K. Primary focal segmental glomerulosclerosis in adults: is the Indian cohort different? Nephrol Dial Transplant 2009;24:3701 - 7.

19. Albaqumi M, Barisoni L. Current Views on Collapsing Glomerulopathy. J Am Soc Nephrol. 2008;19 (7):1276-81.

20. Shetty AA,Tawhari I, Safar-Boueri L, Seif N,Alahmadi A, Gargiulo R et.al. COVID-19 associated glomerular disease. J AM Soc Nephrol. 2021,32(1)33-40.

21. Ray, P. E. , L. Xu , T. Rakusan, and X. H. Liu . A 20-year history of childhood HIV-associated nephropathy. Pediatr Nephrol 2004. 19:1075 - 1092.

22. Ross MJ, Klotman PE. HIV-associated nephropathy. AIDS 2004. 18:1089 - 1099.

23. Gbadegesin R,1, Peter Lavin P,2 Foreman J,1 and Winn M. Pathogenesis and therapy of focal segmental glomerulosclerosis: an update. Pediatr Nephrol. 2011; 26(7):1001 - 1015.

24. Albaqumi M, Barisoni L. Current views on collapsing glomerulopathy. J Am Soc Nephrol. 2008;19(7):1276-81.

25. Choi MJ. Histologic Classification of FSGS: Does Form Delineate Function? CJASN 2013; 8(3): 344-346

26. Arias LF, Franco-Alzate C, Rojas SL. Tip variant of focal segmental glomerulosclerosis: outcome and comparison to 'not otherwise specified' variant. Nephrol Dial Transplant 2011;26:2215 - 2221.

27. Yongzhong Zhong, Feng Xu, Xiaomei Li, Hao Chen, Shaoshan Liang, Xiaodong Zhu, Zhihong Liu and Caihong Zeng. The evolution of morphological variants of focal segmental glomerulosclerosis: a repeat biopsy-based observation. Nephrol. Dial. Transplant. 2016 Jan;31(1):87-95

28. Howie AJ, Brewer DB: Further studies on the glomerular tip lesion: Early and late stages and life table analysis. J Pathol 1985;147: 245 - 255.

29. Micky J. Chun, Stephen M. Korbet, Melvin M. Schwartz and Edmund J. Lewis. Focal Segmental Glomerulosclerosis in Nephrotic Adults: Presentation, Prognosis, and Response to Therapy of the Histologic Variants. JASN, 2004;15(8):2169-2177.

30. Shi SF, Wang SX, Zhang YK, Zhao MH, Zou WZ. Clinicopathologic study of different variants of focal segmental glomerulosclerosis. Zhonghua Bing Li Xue Za Zhi. 2007;36:11-4.

31. Stokes MB, Valeri AM, Markowitz GS, D'Agati VD. Cellular focal segmental glomerulosclerosis: Clinical and pathologic features. Kidney Int. 2006;70:1783-92.

32. Nair R. Focal segmental glomerulosclerosis: Cellular variant and beyond. Kidney Int. 2006;70:1676-8. 